\title{
Ueber die Miliartuberculose.
}

\section{Von Prof. Dr. Hugo Ribbert in Bonn.}

Im 149. Bande von Virchows Archiv hat mein Schüler o. Wild die Lehre Weigerts von der Entstehnng der Miliartuberculose durch plötzlichen Einbruch zahlreicher Bacillen in den Blutstrom angegriffen. Weigert ${ }^{1}$ verteidigte bald darauf seinen Standpunkt, ich ${ }^{2}$ ) entgegnete ihm und kam auch später noch einige Male darauf zurück.

Meine und Wilds Auseinandersetzungen haben lebhafte Opposition hervorgerufen. Es wurden zahlreiche Untersuchungen zur Widerlegung meiner Bedenken und zur Stütze der Weigertschen Lehre vorgenommen, die so vortrefflich erdacht war und wegen ihrer schematischen Einfachheit großen Anklang gefunden hatte. Eine Fülle neuer Beobachtungen trat zutage und erweiterte unsere Kenntnisse der Miliartuberculose beträchtlich. So hat mein Einspruch anregend und fördernd gewirkt und daraus, daß so viele Arbeiten zu seiner Erörterung nötig wurden, geht schon allein hervor, daß er nicht völlig unberechtigt war. Aber damit kann ich mich nicht zufrieden geben, ich muß meine Bedenken in den wesentlichsten Punkten aufrecht erhalten, gebe aber gern zu, daf ich auf Grund jener Arbeiten meine Anschauung in manchen Punkten modifiziert habe, sodaß sie nun vielleicht für eine Verständigung besser geeignet ist.

Zweierlei war es, was mich hauptsächlich zum Widerspruch gegen Weigert veranlaßte. Erstens hob ich hervor, daß die Einbrüche, aus denen die massenhaften Bacillen stammen sollten, sehr oft nicht gefunden würden und daß sie, wenn man sie findet, meist nicht so beschaffen sind, dab sie für eine plötzliche Ueberschwemmung des Blutes ausreichen. Zweitens betonte ich, daß die miliaren Knötchen nicht alle gleich grob, nicht alle gleich alt sind. Wurde es mir so wahrscheinlich, daß die Bacillen nicht auf einmal in den Kreislauf gekommen sind, so mußte ich ihre Vermehrung im übrigen Körper annehmen. Ich schloß daher, daßs sie entweder im Blute oder in den Organen wucherten. Die erstere Möglichkeit mußte ich freilich aufgeben, da die Bakterien in strömenden Flüssigkeiten nicht wachsen. Auch die Annahme, daf die Vermehrung in Capillaren, z. B. der Niere, erfolgen könnte, ließ sich nicht verallgemeinern. D)agegen machte ich ${ }^{3}$ ) auf das Vorhandensein von Gefäßwandtuberkeln in den Lungengefäßien aufmerksam, in denen eine Zunahme der Bacillen vor sich geht. Aber diese Erklärung fand ebensowenig wie jene Einwände Gnade vor den Augen der Anhänger Weigerts. Ich bin daher genötigt, auf die einzelnen Punkte auf Grund fortgesetzter Untersuchungen noch einmal einzugeherl.

Was nun zunächst die Einbruchstellen angeht, so muß ich ihr häufiges, ja fast regelmäfiges Vorkommen heute zugeben. Dagegen kann ich für die meisten Fälle auch jetzt noch nicht anerkennen, daß sie eine ausreichende Quelle zahlloser, mit einem Schlage in das Blut gelangender Bacillen sind. Gewöhnlich handelt es sich um flache, mehr oder weniger rauhe, meist nur leicht unebene, kleinere oder größere Hervorragungen in das Gefäßlumen. Ich führe als Beispiele einige Beschrei-

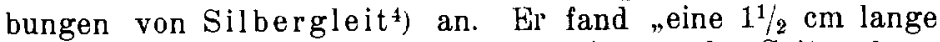
gelbliche Hervorragung der Wand", „eine an der Spitze deutlich ulcerierte Erhebung“, „längliche Hervorragungen mit etwas unebener Oberfläche*, "längliche, stellenweise käsige Verdickung“, „zwei längliche Verdickungen, die eine größtenteils verkäst", "eine Erhebung mit rauher Oberfläche" etc. Wie

1) Diese Wochenschrift 1897, No. 48. - 2) Ibidem 1897, No. 53. - 3) Ueber die Ausbreitung der Tuberculose in Körper. Universitätsprogramm. Marburg 1900. 4) Virchows Archiv Bd. 179. 
sollen aus solchen Herden plötzlich oder in ganz kurzer Zeit zahllose Bacillen in das Blut gekommen sein? Offenbar stellt man sich vor, daß an jeder dieser Erhebungen etwas Aehnliches geschehen ist, wie das, was Schmorl'1) und nach ihm Silbergleit in der Leiche nachahmten, als sie eine weiche Prominenz anstachen und nun aus ihr den breiigen Inhalt sich entleeren sahen. Aber eine derartige Erweichung, die in der Tat regelmäßig vorhanden sein müßte, wenn man an Weigerts Lehre festhalten will, ist doch nur sehr selten nachweisbar. Nun wird man vielleicht sagen, sie könnte ursprünglich bestanden haben. Aber das ,können" genügt nicht, wir verlangen Beweise. Man müßte doch mindestens die zerrissenen Wandungen der erweichten Teile noch auffinden können. Oder sind die nachträglich abgerissen und so völlig entfernt worden, daß nur eine rauhe Fläche zurückblieb? Wie sollte das möglich sein? Dazu gehört ein starker Glaube. Es läßt sich daher zwar für einzelne Fälle vermuten, aber für die weitaus meisten nicht entfernt annehmen, geschweige denn beweisen, daß eine plötzliche Einschwemmung von Bacillen an einer erweichten Stelle stattgefunden hat. Mit einer rauhen Fläche, mit freiliegendem Käse etc. läßt sich nichts anfangen. Von solchen Stellen kann wohl nach und nach immer wieder etwas abgeschwemmt werden, aber von einem massenhaften Uebertritt ist keine Rede. Zudem sind häufig die lokal nachweisbaren Bacillenmelıgen gering. Es ist aber willkürlich, zu behaupten, dab sie friiher reichlicher da waren und fortgeschwemmt wurden. Zuweilen allerdings sieht man viele Bacillen, aber dann sind sie eben noch an Ort und Stelle, und es ist wiederum ohne Berechtigung, dab früher schon auf einmal ähnliche Mengen fortgeschwemmt worden seien. Man sieht also, wie überraschend ungenügend die Anhaltspunkte für den plötzlichen Einbruch massenhafter Bacillen sind. Von einer allein entscheidenden exakten Beweisführung kann nicht gesprochen werden.

Das alles gilt zunächst nur für die Venentuberkel. Silbergleit führt abel auch einen kirschgroßen Tuberkel an, der das Endocard des rechten Ventrikels vorwölbte und erbsengroß durchgebrochen war. Soll aus dem Käse auf einmal eine große Menge von Bacillen ins Blut gekommen sein? Von ihm kani doch nur nach und nach etwas abbröckeln. Das gilt auch für eine „kässige, etwas über erbsengrobe Erhebung von rauher Oberfläche" der Pulmonalarterie. Es gilt aber noch weit inehr für alle Fälle von Tuberculose des Ductus thoracicus. In ihm ist ein plötzlicher Einbruch überhaupt kaum denkbar. In einem Blutgefäß mit seiner abwechselnden Kontraktion und Erschlaffung und seinem kräftigen Blutstrom kann ein erweichter Herd einreißen, im Ductus thoracicus ist das nicht zu erwarten. Der Herd wird langsam usuriert werden und langsam seinen Inhalt an die Lymphe abgeben. Wird aber Käse ohne Erweichung nach und nach freigelegt, wie es in den meisten in der Literatur angeführten Fällen zutrifft, dann werden nur sehr allmählich Bacillen in die Lymphe gelangen, niemals so viele, dab sie allein auf einmal eine Miliartuberculose hervorrufen könnten. Der plötzliche Einbruch in das Gefäßsystem ist demnach für die meisten Fälle theoretisch konstruiert, nirgendwo sicher nachgewiesen und überall unwahrscheinlich.

Aber man kann auch aus anderen L'mständen nachweisen, daß dieser Einbruch meist keine Rolle spielt. Ich meine die verschiedene Größe der Tuberkel. Doch habe ich hier nicht die von mir hervorgehobene Differenz zwischen Spitze und Basis im Auge. Ihre Bedeutung könnte ja durch die Behauptung in Frage gestellt werden, daß die Knötchen überall gleich alt und an der Spitze lediglich rascher gewachsen und deshalb umfangreicher geworden seien. Ich darf aber hier wohl einschalten, daß die Größendifferenz selbst völlig auber Zweifel steht. Es gibt allerdings Fälle, in denen die Tuberkel überall ungefähr gleich umfangreich sind, und solche hat v. Hansemann ${ }^{2}$ ) offenbar gemessen. Aber sie sind in der Minderzahl. in den meisten Fällen sind die Knötchen deutlich verschieden, auch wenn es sich nicht um ältere, sondern um ganz frische Formen handelt. Ja, gerade in dieser kann

1) Münchener medizinische Wochenschrift 1902. No. $33-2$ ) Zentralblatt für pathologisilue Anatomie Bd. 15, S. 257 man den Unterschied manchmal besonders gut feststellen. Man sieht mit blobem Auge in den unteren Teilen der Unterlappen kaum die ersten Spuren der Tuberkel, während sie in der Spitze bereits deutlich hervortreten.

Aber es ist leicht festzustellen, dal die Differenzen im allgemeinen mehr in die Augen fallen, wenn die Lunge mit größeren Knötchen durchsetzt ist. Doch nicht deshalb, weil in ihnen genetisch andere Dinge vorliegen. Denn es handelt sich in solchen Fällen, so auch in dem von mir (l. c.) abgebildeten, von v. Hansemann nicht als Miliartuberculose anerkannten, lediglich darum, daf die Erkrankung langsamer verlief und daß die Tuberkel daher umfangreicher werden konnten. Ihre Vergrößerung schritt dabei um so rascher fort, je näher sie sich der am meisten disponierten Lungenspitze entwickelten. Im übrigen gibt es zwischen der akuten Miliartuberculose und den chronischeren Formen keinen prinzipiellen Unterschied. Es handelt sich nur um verschiedene Stadien. Die Knötchen bestehen in weitaus den meisten Fällen zu allen Zeiten aus einer Gruppe exsudaterfüllter Alveolen und interstitiellen, mehr oder weniger entwickelten Proliferationsprozessen. Sie vergrößern sich durch Ergriffenwerden neuer Alveolen, durch Fortschreiten in die Bronchiolen und durch Ausbreitung der bindegewebigen Neubildung. Reine Granulationsknötchen sind die miliaren Tuberkel selten und niemals in den jüngsten Entwicklungszuständen, sondern nur in chronisch verlaufenden Fällen, in denen die Exsudation gering ist und nach und nach durch die interstitielle Wucherung ersetzt wird.

Theoretisch betrachtet, könnte die Miliartuberculose sehr wohl in eine chronische Lungentuberculose übergehen. - Wenn das für gewöhnlich nicht geschieht, so liegt es daran, daß die Individuen mit Miliartuberculose wegen zu großer Ausdehnung der Erkrankung zu früh zugrunde gehen. Aber hämatogene Tuberculose tritt nicht immer in Form ausgedehnter Miliartuberculose auf, sie kann sich auch auf den mehr disponierten Oberlappen oder nur auf dessen oberen Abschnitt oder überhaupt auf einzelne Herde beschränken und dann zur chronischen Lungenphthise werden. Die Möglichkeit eines derartigen Vorganges wird ja wohl auch allgemein anerkannt, aber man meint meist, die hämatogene Genese sei nicht häufig. Ich habe mich mehrfach ${ }^{1}$ ) in anderem Sinne ausgesprochen, will aber hier nicht weiter darauf eingehen.

Die Verschiedenheit der Knötchen zwischen Spitze und Basis wollte ich indessen, wie gesagt, hier nicht in den Vordergrund stellen. Ich habe mich aber mittlerweile überzeugt, daf in allen Lungenabschnitten neben den gröferen oben und den kleineren unten gewöhnlich noch jüngere Knötchen vorhanden sind, die man mit bloßem Auge leicht übersieht. Man findet die ersten kleinen Gruppen exsudaterfüllter Alveolen mit noch geringer interstitieller Wucherung. Man sieht gröbere, oft schon zentral verkäste, daneben die ältesten, meist riesenzellenhaltigen Tuberkel, die das makroskopische Bild beherrschen. Selbstverständlich muß man diese Untersuchungen an Serienschnitten vornehmen, da man sonst hei kleineren Herdchen nicht wissen kann, ob man nicht die Randteile der größeren vor sich hat. Auch mub man nicht zu alte Fälle in Betracht ziehen. Denn wenn alle Knötchen schon einige bis viele Wochen bestanden haben, dann verwischen sich die Unterschiede. Läßt man aber diese beiden Punkte nicht aufer acht, dann wird man das verschiedene Alter leicht feststellen können. Ich werde sogleich noch eine ähnliche Erfahrung mitteilen, die sich auf die Intimatuberkel bezieht. Wenn nun auf der einen Seite die Tuberkel nicht alle zugleich, sondern während eines längeren Zeitraums nacheinander zustande gekommen sind und wenn anderseits die GefäBwandtuberculosen meist nicht eine Quelle für eine einmalige Ueberschwemmung des Blutstroms sein können, so bleibt nur die Möglichkeit, daß die Bacillen immer wieder aufs neue in den Kreislauf gelangten und sich in den Organen festsetzten. Wo sind nun die Stellen zu suchen, von denen aus das geschah? Ich habe schon früher darauf hingewiesen ${ }^{2}$ ), daf, was man bis dahin nicht wubte, in den Gefäfen, besonders den

1) Zuletzt in dieser Wochenschrift 1902, No. 17. - 2) 1. c. Universitätsprogramm 
Arterien der Lunge sehr viele Intimatuberkel vorkommen, in denen mehr oder weniger zahlreiche Bacillen vorhanden sind, aus denen sie also auch in das Blut übertreten können. Das ist denn auch von anderen Seiten bestätigt worden, aber man hat bestritten, dal diese Gefäßwandprozesse regelmäßig oder häufig seien und dab sie zur Erklärung ausreichten. Ich muß daher das Resultat fortgesetzter Untersuchungen mitteilen, die mir lediglich eine Bestätigung meiner damaligen Befunde lieferten.

Aber ich muß zunächst hervorheben, daf die Aufsuchung der Intimatuberkel mit Sicherheit nur an Serienschnitten gelingt, in denen man ein Gefäb auf längere Strecken und eventuell mit Verzweigungen verfolgen kann. Und zweitens mul ich darauf hinweisen, daf nur wenig vorgeschrittene, durch feine Knötchen ausgezeichnete Miliartuberculosen vollen Erfolg versprechen. Je länger der Prozeb dauert, desto mehr wird durch Vergrößerung der Intimaherdchen das Gefäßlumen verlegt, und wenn das geschehen ist, dann fehlt eben das charakteristische Bild der in das Lumen hineinragenden Prominenz. Geht man unter Berücksichtigung dieser Bemerkungen an die Untersuchung, so wird man die Intimatuberkel nie vermissen, man wird sie im Gegenteil sehr häufig antreffen. Es ist für jeden leicht, sich von ihrer Gegenwart zu überzeugen. Deshalb verzichte ich auf Abbildungen und verweise ihretwegen auf meine Programmschrift (l. c.) und auf meine spezielle pathologische Anatomie (Fig. 271).

Die lntimatuberkel kann man manchmal in einer längsdurchschnittenen Arterie zu zweien oder mehreren in demselben Gesichtsfeld nachweisen, oder man sieht in eeinem quergetroffenen, durch eine Reihe von Schnitten verfolgbaren Gefäß mehrere hintereinander auftauchen. Nicht selton liegen sie zu zweien nebeneinander oder an gegenüberstehenden Flächen der Gefäßwand, sodaß man an eine Ueberimpfung von einer zunächst beteiligten Stelle auf eine benachbarte schließen kann. Ihre Zahl ist oft sehr beträchtlich. Ich habe z. B. in 16 aufeinander folgenden Schnitten von $8 \mathrm{~mm}$ im Quadrat 22 Intimatuberkel gezählt. Danach müssen in einer Lunge bei Miliartuberculose unzählige, mindestens aber viele Tausende vorhanden sein. Daß solche ungeheuren Mengen von Intimatuberkeln viele Bacillen an das Blut abgeben können, ist selbstverständlich.

Sie bieten aber noch einen bemerkenswerten Befund. Sie sind nämlich durchaus nicht alle von gleichem Alter. Man findet solche, die zentral verkäst sind und im übrigen aus jugendlichem, zellenreichem Gewebe, zuweilen mit Riesenzellen, bestehen, man bemerkt andere, die noch nichts weiter darstellen als kleine Höckerchen aus thrombotischem Material, wieder andere, deren Organisation beginnt oder schon weiter vorgeschritten ist, kurzum alle möglichen Altersdifferenzen. Also auch die Intimatuberkel sind durch eine über längere Zeit sich erstreckende, immer erneute Ansiedelung von Bacillen entstanden. Sie stimmen hierin demnach mit den übrigen Tuberkeln überein und beweisen mit ihnen gemeinsam aufs neue die allmähliche Entstehung der Miliartuberculose. Für sie spricht auch noch der Umstand, dab bei Individuen, die an Miliartuberculose erkrankten, bei denen also schon viele Knötchen in allen Organen vorhanden sind, Bacillen mit Leichtigkeit im Blute nachgewiesen wurden. Sie können nicht wohl von dem ersten Einbruch herrühren, selbst wenn man diesen als plötzlich und reichlich voraussetzt. Denn wir wissen, wie schnell in das Blut gebrachte Bakterien aus ihm verschwinden. Sie müssen vielmehr immer wieder aufs neue hineingekommen sein und werden sich auch immer wieder hier oder dort festsetzen und die jüngeren Tuberkel erzeugen. Von der Genese der Miliartuberculose durch einen einmaligen, schnellen Einbruch und eine in kurzer Zeit erfolgende Bildung der Tuberkel kann somit auch aus diesem Grunde keine Rede sein.

Die Intimaprozesse bedeuten eine Endangitis tuberculosa im kleinen. Sie stehen den großen Intimatuberkeln parallel, die man jetzt nach dem Vorgange Bendas als die wichtigsten Quellen der Bacillen ansieht. Man hat also die Vorstellung Weigerts, derzufolge es sich hauptsächlich um einen Einbruch extravaskulärer tuberculöser Herde in das Ge-
fäBsystem handeln sollte, wesentlich eingeschränkt, Dazu hat besonders mein Einspruch gegen Weigerts Lehre beigetragen. Sie konnte nur noch in der durch die Auffindung der Intimatuberkel modifizierten Form festgehalten werden. Wie nun aus diesen GefäBherden, so können auch aus den kleinen Intimaknötchen Bacillen an das Blut abgegeben werden. Und wenn auch jedes einzelne nur wenige abgibt, so ist doch ihre Gesamtmenge beträchtlich.

Aber nachdem Bacillen aus dem primären Einbruch in das Blut gelangt sind, wird dann ein weiterer Uebertritt nicht nur aus den kleinen Intimatuberkeln stattfinden. Denn es ist leicht einzusehen, daß in den Fällen, in denen wirlilich eine plötzliche Eröffnung eines erweichten Herdes stattgefunden hat, aus diesem auch weiterhin noch Bacillen in die Zirkulation gelangen müssen.

Die Entleerung des Herdes kann ja nicht auf einmal stattfinden. Es entsteht vielleicht zunächst eine kleine Oeffnung, aus der eine gewisse Menge Brei hervoriluillt. Langsam wird durch den Blutdruck noch weiterer Inhalt aus. gepreßt, langsam erweitert sich die Oeffnung und der eintretende Fliissigkeitsstrom spült allmählich noch melır erweichtes Material aus. Das alles geht im Laufe von Tagen und sicherlich auch Wochen vor sich. Wie hat man nur an dem Gedanken einer einmaligen Ausspülıng festhalten könnell? Man sehe sich die atheromatösen Defekte der Aorta an, in denen trotz des energischen Blutstromes noch reichlicher cholestearinhaltiger Brei gefunden wird, der hier sicher schon seit Monaten liegt und nur ganz allmählich sich vermindert. Diese Ueberlegungen gelten aber nur für die seltenen Fälle rascher und ausgiebiger Einbrüche. Meist handelt es sich $11 \mathrm{~m}$ ein langsameres Defektwerden der tuberculösen Hervorragungen der Intima und demgemäß um ein nach und nach erfolgendes Abspülen und Abbröckeln der erweichenden, bacillenhaltigen Massen. Dabei wird das Blut über längere Zeit hin immer wieder mit Bacillen versehen, die nun jene miliaren Intimatuberkel erzeugen.

Aber noch eine Ueberlegung muß hinzukommen. Wenn wir gezwungen sind anzunehmen, daß die Bacillen nicht aut einmal ins Blut einbrechen, so bleibt die Frage offen, weshalb denn die Miliartuberculose sich nicht häufiger an die Gegenwart von Bacillen im Blut anschließt. Sie gelangen ja doch bei Phthisikern und anderen Tuberculösen nicht selten in den Kreislauf.

Da ist denn zunächst zu betonen, daß der Umfang der tuberculösen Ansgangsprozesse in der Tat eine Rolle spielt. Wenn bei Tuberculose der Lungen alls kleinen, makroskopisch nicht präparierbaren Gefäßen einzelne Bacillen austreten, so werden sie nur spärliche Intimatuberkel erzeıgen, in deneı insgesamt thre Vermehrung so geringfügig bleibt, daf eine allgemeine Miliartuberculose nicht entsteht, und das um so weniger, als auch aus jenen kleinen Einbrüchen weiterhin nur wenig Bacillen geliefert werden. Es entstehen abel iminerhin manchmal sekundäre Tuberkel der übrigen Organe, der Venen, Placenta, Lunge etc. Mit der steigenden Menge der Bacillen werden auch diese Herde reichlicher und ebenso die miliaren Intimatuberkel, aus denen die Bacillen immer wieder ins Blut zurückgelangen. Derartige größere Mengen werden aber natürlich besonders aus umfangreicheren Gefäßherden stammen, und insofern ist die Miliartuberculose von deren Existenz gewöhnlich abhängig.

Aber auch bei dieser Erklärung nehme ich an, daß die Miliartuberculose vor allem bei den Individuen entsteht, die dell Bacillen aus irgend einem Grunde besonders günstige Entwicklungsbedingungen bieten. Nur muß man sich nicht vorstellen, daß diese Bedingungen erst im Zeitpunkt des Einbruches zur Geltung kämen. Im Gegenteil wird es so sein, daß schon die Bildung der grofien Intimatuberkel auf der Grundlage der Disposition zustande kommt. So würde es sich erklären, dab man dio Endangitis tuberculosa bei Phthisikern im allgemeinen nicht antrifft, obgleich doch bei ihnen oft Bacillen im Kreislauf sind. Auch das Einwachsen verkäsender Lymphdrüsen in Venen und in den Ductus thoracicus wird darauf beruhen, daß die befallenen Individuen auf Grund unbekannter Umständo die lokale Tuberculose ausgiebiger als weniger disponierte zum 
Fortschreiten brachten. Die einmal vorhandene Disposition wird dann auch die dauernde Vermehrung der Bacillen in den defekten Gefäßherden und in den miliaren Intimatuberkeln begünstigen.

Nun interessiert uns schlieblich noch die Frage, weshalb die miliaren Tuberkel in der Junge so ganz gleichmäßig verstreut sind. Man könnte doch denken, dab die Bacillen sich überall festsetzten und eine diffuse Verdichtung erzeugten. Ich habe früher diesen Befund daraus abzuleiten versucht, daf die Bacillen sich in den lymphatischen Herdchen ${ }^{1}$ ) oder in den feinsten Bronchiolen festsetzen. Ohne diese beiden Erklärungen völlig fallen zu lassen, gebe ich jetzt einer dritten den Vorzug.

Die Tuberkel entstehen meist von den letzten Enden der Pulmonalarterie aus kurz vor der Teilung in die zu den Alveolen fihrenden Aeste. Bei frischen Miliartuberkeln kann man sich, freilich nur bei Anwendung von Serienschnitten, ohne Mühe iiberzeugen, daß in die Knötchen ein Arterienast hineingeht, dah er innerhalb derselben durch Thrombose oder Arterienwucherung verschlossen ist und dab er dann wieder ungeteilt oder nach Zerlegung in Aeste hervortritt. Es unterliegt danach keinem $Z$ weifel, daß die Bacillen sich in den letzten Pulmonalarterienästen. ähnlich wie in den etwas größeren Zweigen, in der Intima festsetzen. Während sie aber in letzteren Thrombosen und Wucherungen veranlassen, die in das weitere Lumen knötchenförmig prominieren, führen sie in den ferneren Kanälen rasch zur Obliteration, sodaß man hier die Intimaveränderungen nur in den frühesten Stadien als Vorsprünge in das Lumen wahrniunmt, während man später die verschlossenen Gefäße in den um sie entstehenden Knötchen nicht mehr ohne weiteres erkennt. Da aber diese engen Arterien neben den lymphatischen Herdchen oder durch sie verlaufen, ist die Lokalisation der Tuberkel wiederum dieselbe wie nach jenen beiden ersten Annahmen. So erklärt sich die gleichmäßige Verteilung der Lungentuberkel.

Für die Vermehrung der Bacillen und deren erneuten Uebertritt ins Blut kommen aber die Gefäße im Innern der Knötchen nur wenig in Betracht. Denn sie werden zu schnell verschlossen, als daß der Blutstrom aus ihnen etwas ausspülen könnte. Nur wo die Intimatuberkel in fliefendes Blut hineinragen, können sie Bacillen abgeben.

Zusammenfassend bleibe ich also dabei, daß die Miliartuberculose meist nicht durch eine einmalige Ueberschwemmung des Blutes mit Bacillen zustande kommt. Sie entsteht vielmehr dadurch, daß geringere Mengen in den Kreislauf gelangender Bacillen sich nachträglich, vor allem in den miliaren Intionabaeillen, vermehren und daß andere Bacillen immer aufs neue aus den primären Einbruchstellen in die Zirkulation übertreten. 